\title{
1 Epitaxial graphene homogeneity and quantum Hall 2 effect in millimeter-scale devices
}

3 Yanfei Yang ${ }^{\dagger *}$, Guangjun Cheng ${ }^{\dagger}$, Patrick Mende ${ }^{\ddagger}$, Irene G. Calizo ${ }^{\dagger, \S}$, Randall M. Feenstra ${ }^{\ddagger}$,

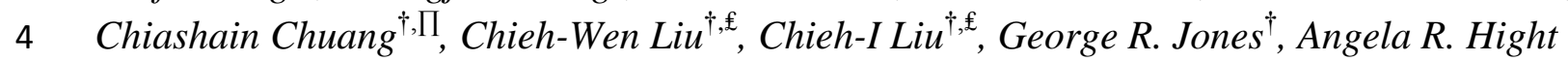

$5 \quad$ Walker ${ }^{\dagger}$, and Randolph E. Elmquist

6 'National Institute of Standards and Technology, Gaithersburg, MD 20899-8171, USA

$7 \quad{ }^{ \pm}$Department of Physics, Carnegie Mellon University, Pittsburgh, PA 15213-3890, ${ }^{1}$ USA

$8 \S$ Present address: Mechanical and Materials Engineering, Florida International University, 9 Miami, FL 33174

$10 \Pi$ Present address: Graduate School of Advanced Integration Science, Chiba University, Chiba, 11 263-8522, Japan

$12{ }^{£}$ Graduate Institute of Applied Physics, National Taiwan University, Taipei 106, Taiwan

13 *E-mail: yanfei.yang@ nist.gov

14 KEYWORDS: epitaxial graphene, transport mobility, carrier density, adsorption-induced molecular doping, strain, low-energy electron microscopy, Raman microscopy, quantized Hall 16 effect

17 ABSTRACT: Quantized magnetotransport is observed in $5.6 \times 5.6 \mathrm{~mm}^{2}$ epitaxial graphene devices, grown using highly constrained sublimation on the $\mathrm{Si}$-face of $\mathrm{SiC}(0001)$ at high temperature $\left(1900^{\circ} \mathrm{C}\right)$. The precise quantized Hall resistance of $R_{x y}=\frac{h}{2 e^{2}}$ is maintained up to record level of critical current $I_{x x}=0.72 \mathrm{~mA}$ at $T=3.1 \mathrm{~K}$ and $9 \mathrm{~T}$ in a device where Raman microscopy reveals low and homogeneous strain. Adsorption-induced molecular doping in a second device reduced the carrier concentration close to the Dirac point $\left(n \approx 10^{10} \mathrm{~cm}^{-2}\right)$, where mobility of $18760 \mathrm{~cm}^{2} / \mathrm{Vs}$ is measured over an area of $10 \mathrm{~mm}^{2}$. Atomic force, confocal optical, and Raman microscopies are used to characterize the large-scale devices, and reveal improved

$25 \mathrm{SiC}$ terrace topography and the structure of the graphene layer. Our results show that the

*Corresponding authors: Tel: +1 3019754421 (Yanfei Yang); +1 3019756591 (Randolph Elmquist). E-mail addresses: yanfei.yang@nist.gov (Yanfei Yang); randolph.elmquist@nist.gov (Randolph Elmquist). 
structural uniformity of epitaxial graphene produced by face-to-graphite processing contributes

27 to millimeter-scale transport homogeneity, and will prove useful for scientific and commercial applications.

\section{INTRODUCTION}

Wafer-scale monolayer graphene [1,2] can be produced by thermal decomposition of certain polytypes of silicon carbide [3] (SiC) or by chemical vapor deposition (CVD) on metal catalyst substrates [2]. While CVD graphene forms randomly oriented domains to match the crystal orientation of the metal catalyst, epitaxial graphene (EG) forms a single domain on monocrystalline wafers of hexagonal $\mathrm{SiC}(0001)$ [4] and the insulating $\mathrm{SiC}$ substrate is immediately suitable for fabrication of electronic [5], plasmonic[6] and photonic[7] devices. Quantum Hall effect (QHE) standards produced from EG [8-9] can be operated economically at lower magnetic fields and higher temperatures than GaAs-AlGaAs heterostructures [10]; thus EG devices are likely to become the premier source of resistance traceability in practical metrology and their optimization is of great interest to the electrical metrology community.

Efforts to produce nearly defect-free monolayer EG on SiC generally involve control of the high-temperature vapor phase. For example, annealing in atmospheric-pressure Ar gas [11] or in a small confining enclosure [12] helps to raise the partial pressures of sublimated $\mathrm{Si}, \mathrm{Si}_{2} \mathrm{C}$ and $\mathrm{SiC}_{2}$ closer to equilibrium at high temperature, and the number of defects in graphene is then reduced and the morphology of vicinal $\mathrm{SiC}(0001)$ surfaces is generally improved. However, dissociated carbon atoms may diffuse anisotropically [13], leading to the formation of multiple graphene layers near the edges of the terraces [14]. Furthermore, SiC restructuring [3] and energetically-favorable step-bunching also may produce undesirable terrace facet edges $[14,15]$ that face off-axis by $\approx 30^{\circ}$ on vicinal $\mathrm{SiC}(0001)$. Atomic-layer-resolved characterization has shown significant delamination of the carbon buffer layer $[15,16]$ on facet edges that separate adjacent terraces. Here, we report precision measurements of the QHE in millimeter-scale EG devices at high current and correlate the quantized magnetotransport to microscopy data,

52 including structural reorganization of the $\mathrm{SiC}$ surface, $\mathrm{EG}$ layer number and distribution, and 53 strain as measured by Raman microscopy. 


\section{Sample Preparation}

\subsection{High Temperature EG Growth with Vapor Constraint}

To minimize the possible complications due to the substrate, we produce EG using a constraint on vapor diffusion provided by close proximity to polished pyrolytic graphite substrates (SPI Glas 22). The samples were diced from two $76 \mathrm{~mm} \mathrm{SiC(0001)} \mathrm{semi-insulating}$ wafers (Cree, Inc. ${ }^{* *}$ ) of nominal miscut $0.00^{\circ}$, with sample miscut measured to be $\leq 0.10^{\circ}$ from atomic force microscopy (AFM) images. Samples were rinsed in HF and deionized water before processing, and arranged facing to graphite (FTG) with separation distance limited only by sample and substrate flatness (Fig. 1a). Processing was done in a graphite-lined resistive-element furnace (Materials Research Furnaces Inc.) with heating and cooling rates near $1.5^{\circ} \mathrm{C} / \mathrm{s}$. The initial heating occurs in forming gas $\left(96 \% \mathrm{Ar}, 4 \% \mathrm{H}_{2}\right)$ at $100 \mathrm{kPa}$ with at least $30 \mathrm{~min}$. cleaning of the substrates at $1050{ }^{\circ} \mathrm{C}$, which may serve to hydrogenate the $\mathrm{SiC}$ surface $[17,18]$. The chamber was then flushed with Ar gas, and filled with $100 \mathrm{kPa}$ Ar derived from 99.999\% liquid Ar before annealing at $1900{ }^{\circ} \mathrm{C}$. The annealing process utilized a commercial process controller and a type-C thermocouple located a few $\mathrm{cm}$ above the sample.

Figures $1 \mathrm{~b}$ and $1 \mathrm{c}$ show the optical images of two samples processed with and without FTG configuration, respectively. The sample processed with Si-face open to Ar (Fig. 1c) shows inhomogeneous EG coverage all over the substrate. The FTG sample (Fig. 1b) has uniform EG coverage (grey area) toward its center, while lower vapor pressure near its borders has allowed the formation of thick, graphitic layers (white area). Raman spectra confirm these results and are shown in Figs. 1d-f from five locations along a linear scan of a FTG sample. Both the $\mathrm{G}$ band $\left(\approx 1600 \mathrm{~cm}^{-1}\right)$ and the $\mathrm{G}^{\prime}$ band $\left(\approx 2700 \mathrm{~cm}^{-1}\right)$ from EG evolve with the distance $d$ from the edge and indicate the variation in layer number. Figure 1e magnifies the region of the $G^{\prime}$ band, excluding the peak at $d=20 \mu \mathrm{m}$, and Fig. 1f shows the exponential decrease of the integrated G' peak area toward the center of the substrate. At interior distances $d>\approx 200 \mu \mathrm{m}$, most $\mathrm{G}^{\prime}$ peaks take on the shape of a single Lorentzian with a full width at half maximum $(\mathrm{FWHM})$ of $\approx 40 \mathrm{~cm}^{-1}$, which is the fingerprint for identifying monolayer EG. 

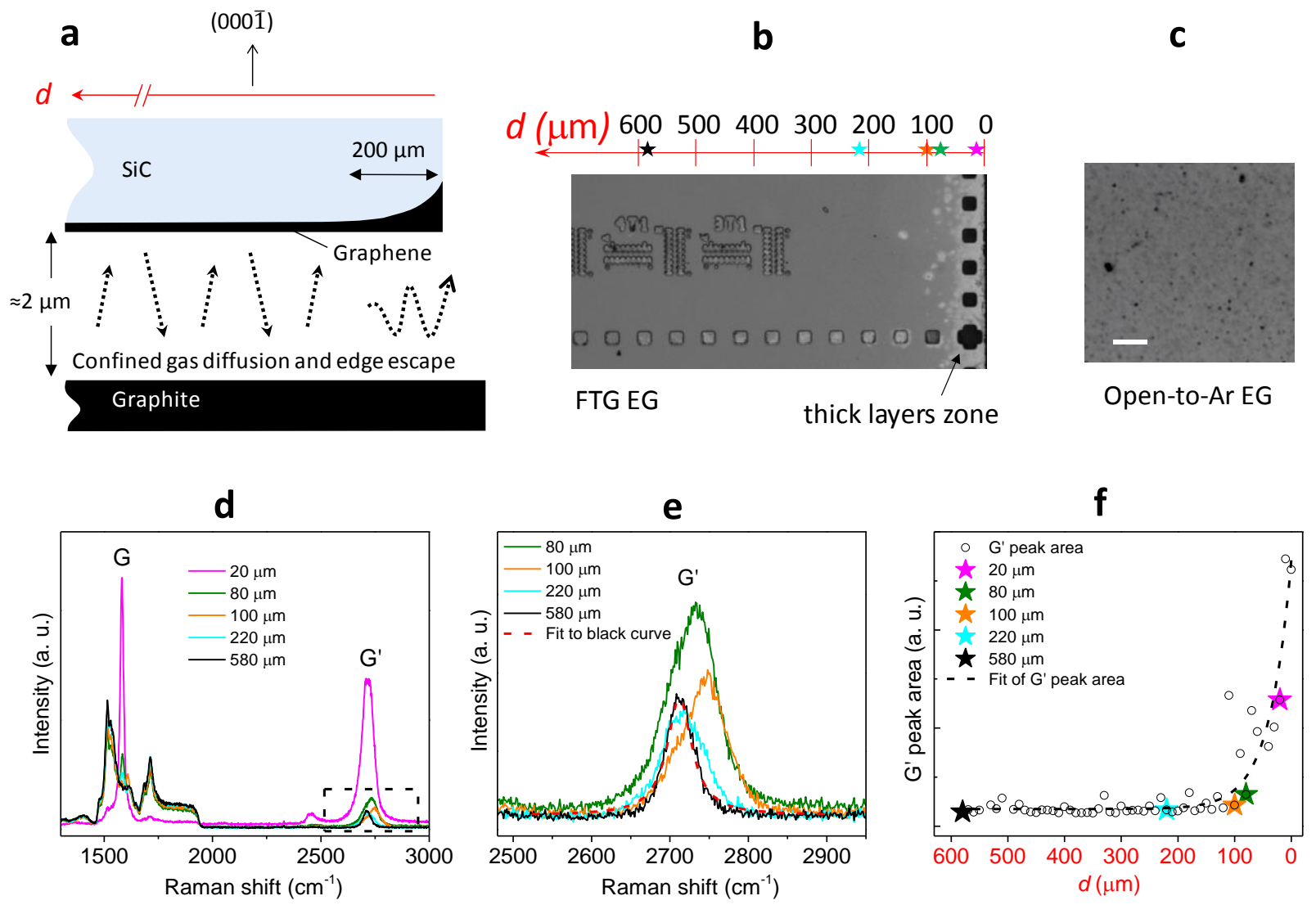

81

Figure 1. (a) Diagram showing a cross-section of the SiC sample and polished graphite during FTG processing. (b) Optical image showing the edge region of a $7.6 \mathrm{~mm}$ square sample processed at $1950{ }^{\circ} \mathrm{C}$ for $1800 \mathrm{~s}$ with FTG configuration. The squares and pattern are fiducials etched into the SiC substrate. (c) Optical image of a sample processed with Si-face open to Ar at $1900{ }^{\circ} \mathrm{C}$ for $210 \mathrm{~s}$, showing the center region with non-uniform graphene. The white scale bar is $10 \mu \mathrm{m}$. (d) Representative Raman spectra vs. distance from the edge of FTG samples, showing G and G' Raman peaks of EG at positions $d=20 \mu \mathrm{m}$ (magenta), $80 \mu \mathrm{m}$ (green), $100 \mu \mathrm{m}$ (orange), $220 \mu \mathrm{m}$ (cyan), and $580 \mu \mathrm{m}$ (black). (e) Zoom-in of Raman G' peak spectra from (b), as indicated by the dashed black box. The G' peak at $d=20 \mu \mathrm{m}$ is omitted for clarity. The G' peak at $d=580 \mu \mathrm{m}$ can be fit well with a single Lorentzian curve as shown. (f) Integrated G' peak area vs. distance from the sample edge. The locations for the data shown in (d)-(f) are marked by colored stars along the axis above the optical image in (b). The data shown in (d)-(f) are marked with the same color scheme. 
In this paper, we will focus on two FTG-grown samples of size $7.6 \times 7.6 \mathrm{~mm}^{2}$. We patterned a semi-octagonal active channel utilizing the center region of the FTG samples, which has an area of $27 \mathrm{~mm}^{2}$ (see the inset of Fig. 4). The edge of the active graphene channel is $1 \mathrm{~mm}$ from the edge of the substrate so that the periphery region of thick layers is avoided. In fabricating the magnetotransport devices, the active EG surfaces were kept uniformly resist-free by depositing $\mathrm{Pd} / \mathrm{Au}$ as a thin layer prior to standard photolithography processing, and afterwards removing the $\mathrm{Pd} / \mathrm{Au}$ layer from the EG region by immersing the devices in dilute aqua regia (by volume, $\mathrm{HNO}_{3}: \mathrm{HCl}: \mathrm{H}_{2} \mathrm{O}=1: 3: 4$ ) for 45 seconds. This fabrication process [19] initiates the attachment of molecular dopants, and can result in carrier concentrations below $n \approx 10^{10} \mathrm{~cm}^{-2}$ in ungated EG samples, compared to as-grown monolayer EG with substrate-induced doping as high as $n \approx 10^{13}$ $\mathrm{cm}^{-2}$. The carrier concentration can be tuned by adding or removing molecular dopant using 106 chemical- or heat-treatment (Fig. S7).

\section{Characterization}

Our present work on devices composed of FTG-grown material shows that the graphene lattice strain and specifically homogeneous low strain is predictive of EG device transport characteristics at millimeter-scale. Exceptional magnetotransport at millimeter-scale was measured in two samples produced using FTG confinement at $1900{ }^{\circ} \mathrm{C}$ for $210 \mathrm{~s}$ (sample A) and 235 s (sample

B). A magnetotransport device of $5.6 \mathrm{~mm}$ in height and width was fabricated at the center of each sample, as shown in the inset of Fig. 2a. The surfaces of both samples were characterized by contrast-enhanced optical imaging [20] (Fig. 2a and Supplement Fig. S1), AFM (Figs. 2b,c), and confocal optical microscopy (Figs. 3a,b).

\subsection{Optical and Atomic Force Microscopy}

The FTG [21] method leads to uniform EG growth with limited terrace restructuring on clean, low-miscut, chemically-mechanically polished $\mathrm{SiC}(0001)$ substrates [22], and often results in crescent-shaped terraces having small areas and low aspect ratios, as shown in Fig. 2. Figure $2 \mathrm{~b}$ shows details of the topographic structure produced by the FTG process at $1900{ }^{\circ} \mathrm{C}$ on the 121 surface of low-miscut $\mathrm{SiC}(0001)$, imaged by AFM, and the corresponding phase image (Fig. 2c) shows uniform contrast. We have found that this terrace topography, together with the near- 
123 equilibrium FTG environment, supports more isotropic carbon diffusion compared to parallel,

124 linear terraces, thus reducing the tendency to form extended bilayer ribbons. Annealing samples

125 at $T>1800{ }^{\circ} \mathrm{C}$ with two $\mathrm{SiC}(0001)$ surfaces arranged face-to-face [22,23] results in uncontrolled

126 step-bunching of the terraces (see Supplement Fig. S2a,b). This large change in substrate

127 topography may be produced by the vapor phase transfer of molecules between the facing

128 samples. Under similar conditions, but annealing the sample facing Ar background gas, it has

129 been shown elsewhere that sub- $\mu \mathrm{m}$ scale pits are likely to develop on low-miscut SiC substrates

130 [24] (see Supplement Fig. S2c). Our results show that FTG confinement reduces structural

131 disorder due to pitting of the SiC surface for low-miscut substrates. Reduced mobility in EG

132 transport has been correlated with the frequency of pitting [24] and with the size and height of 133 underlying $\mathrm{SiC}$ terraces [20,25].

\section{a}

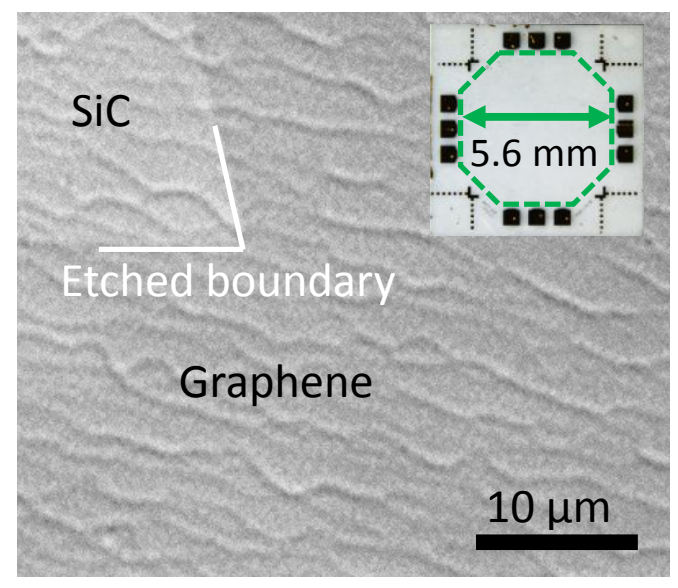

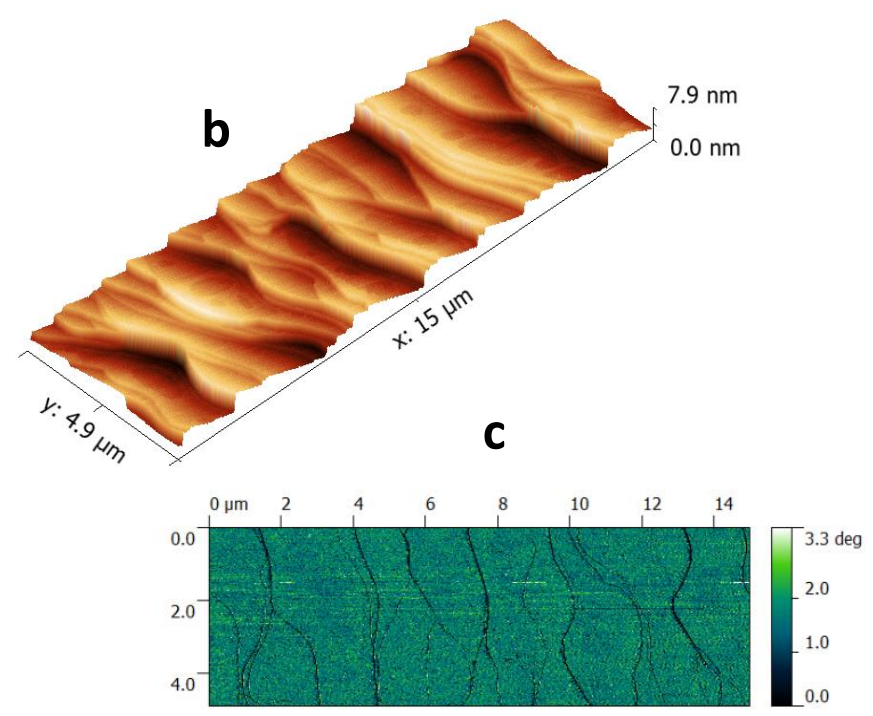

134

135

136

137

138

139

140

141

Figure 2. (a) Optical image of a region of sample A, with enhanced contrast to show where EG has been removed (upper left of image), labeled $\mathrm{SiC}$. Inset shows a large-scale device with twelve symmetric gold contacts. (b) AFM topography image of a FTG sample with step height displayed as contours in the vertical direction. (c) AFM phase image corresponding to the height image in (b).

\subsection{Confocal Laser Scanning Microscopy}

On sample A, the confocal laser scanning microscopy (CLSM) shows a nearly uninterrupted monolayer with only a few multilayer inclusions, which appear as small irregularly-shaped 
142 bright spots or ribbons in reflective confocal microscope images (see Fig. 3a and Supplement

143 Figs. S2d,e). Note the large fiducial marker in the upper right. On sample B, bilayer ribbons and

144 small patches of buffer layer are more common in some regions (Fig. 3b), but still occupy a

145 small percentage of the sample surface. Sample B has low terrace topography, but the terraces

146 are wider and more irregular in some areas (Supplement Fig. S1) indicating isolated step

147 bunching. While $\mathrm{C}$-face $\mathrm{SiC}$ processed with a graphite cap is reported to develop long graphene

148 ribbons [21], we find that both terrace step bunching and multilayer EG growth are much more

149 limited by FTG growth on the Si-face under optimized growth conditions.
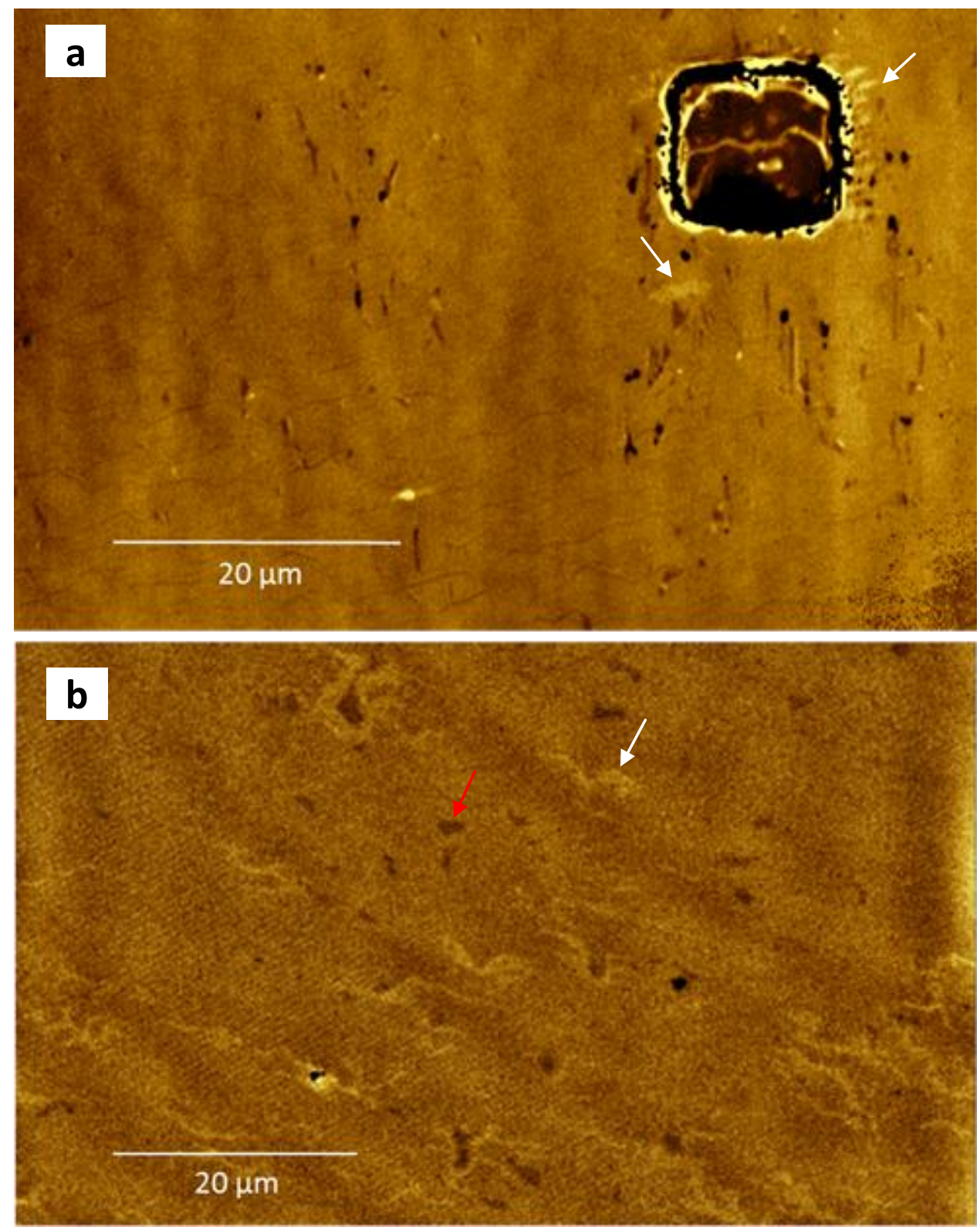
Figure 3. Confocal microscopy images of sample A and sample B. (a) Area of sample A, imaged by contrast-enhanced confocal microscopy with reflected $405 \mathrm{~nm}$ light. Monolayer graphene appears as a uniform background with terraces barely visible. The square region at upper right is a $500 \mathrm{~nm}$ deep etched fiducial mark, showing small fingers of multilayer graphene along its right side, and a bilayer patch just below its lower left corner (indicated by the white arrows). (b) Area of sample B near the left-center contact, imaged by contrast-enhanced confocal microscope with reflected $460 \mathrm{~nm}$ light. Monolayer appears as a uniform background, with buffer layer or no graphene showing as darker patches (indicated by the red arrow) and bilayer or multilayer (indicated by the white arrow) appearing as lighter patches or ribbons.

\subsection{Raman Microscopy}

Raman spectroscopy is a powerful nondestructive technique used to characterize atomically thin graphene samples. The two-phonon $\mathrm{G}^{\prime}$ band of monolayer graphene can be well fit by a single Lorentzian. Furthermore, the position of the $\mathrm{G}^{\prime}$ band has been correlated to the strain in the conductive EG lattice [26-28]. Studies of graphene on SiC also have established that the G' band has only a weak dependence on carrier density [29], that strain can change suddenly where the EG layer crosses a terrace edge [30], and that low and uniform strain is often related to improved transport [31]. Raman data were obtained in closely-spaced grids after transport devices were fabricated on the two samples. No visible D band near $1350 \mathrm{~cm}^{-1}$ in the Raman spectra with two different laser excitation wavelengths (Supplement Fig. S3) indicates lowdefect EG in both samples. Raman maps (see Methods) were generated for three large, wellseparated regions in the device spanning the midline of sample $\mathrm{A}$, across an area of $\approx 5 \mathrm{~mm}$ width (Inset in Fig. 4a). Cross-correlated data for the resulting spectra are shown in Fig. 4a. Of the Raman data collected from the three areas, nearly $99 \%$ of the G' spectra (1178 points) are symmetric and are fit with a single Lorentzian. The fitted $\mathrm{G}^{\prime}$ linewidths are less than $40 \mathrm{~cm}^{-1}$, ranging from $27.5 \mathrm{~cm}^{-1}$ to $38.2 \mathrm{~cm}^{-1}$, and the mean center position is $(2729.7 \pm 2.7) \mathrm{cm}^{-1}$, ranging from $2721.3 \mathrm{~cm}^{-1}$ to $2737.5 \mathrm{~cm}^{-1}$. These closely grouped results with narrow linewidths reveal an unprecedented uniformity for EG, since both strain and layer number variation can affect these fitted parameters. The mean linewidth value $\Gamma_{\mathrm{G}^{\prime}}=(31.7 \pm 1.4) \mathrm{cm}^{-1}$ may be compared to $\Gamma_{\mathrm{G}^{\prime}} \approx 25$ $\mathrm{cm}^{-1}$, ranging from $22 \mathrm{~cm}^{-1}$ to $35 \mathrm{~cm}^{-1}$, as reported recently [32] for exfoliated graphene on $\mathrm{SiO}_{2}$ capped by hexagonal BN (h-BN). 

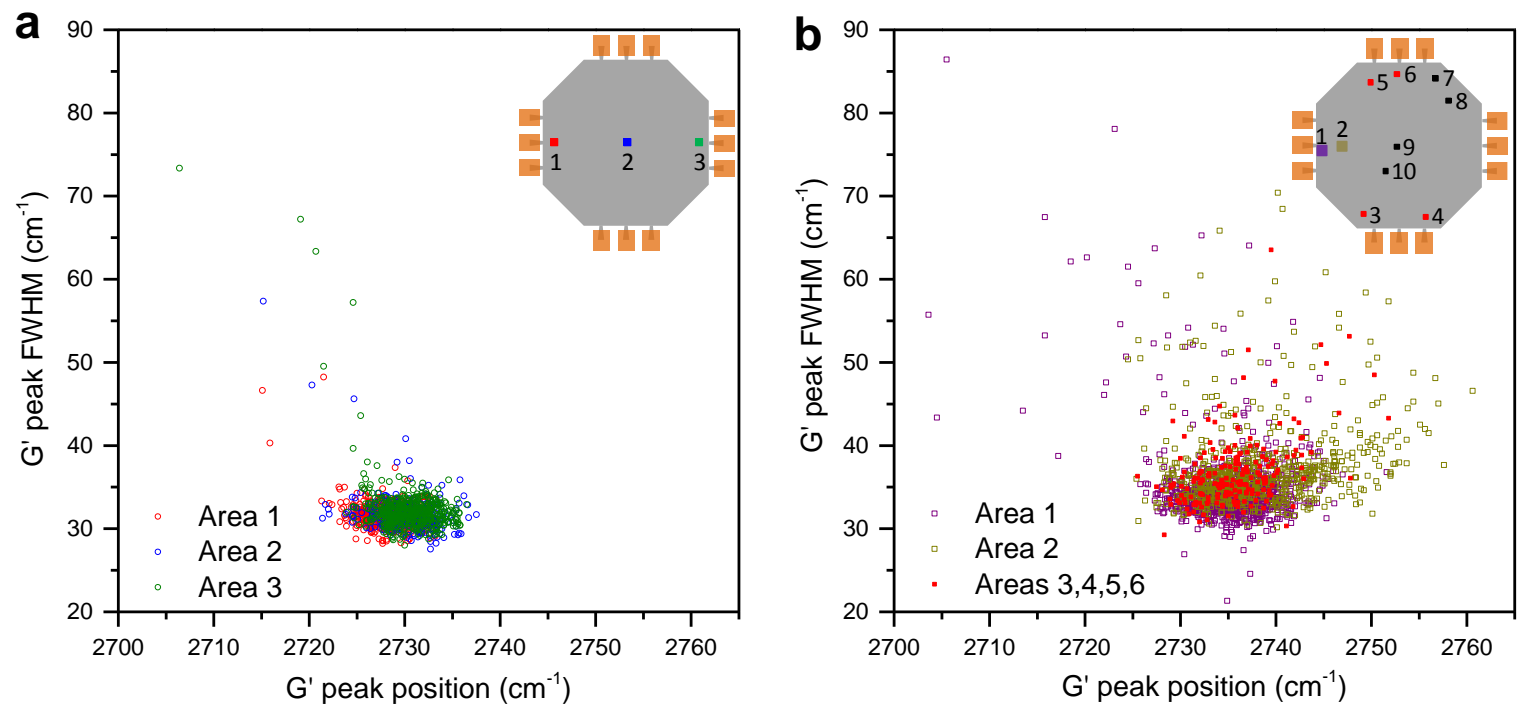

181

182

183

184

185

186

187

188

189

190

191

192

193

194

195

196

197

198

199

Figure 4. Raman G' peak analysis for two octagonal devices. (a) Correlation between FWHM and peak position for G' peaks collected from three areas in sample A, located as shown in the inset using red, blue, and green. (b) Correlation between FWHM and peak position for G' peaks collected from six areas in sample B, located and identified by color as shown in the inset.

The Raman data from sample B (see Fig. 4b) in general have wider distributions, tending toward higher values of $\Gamma_{\mathrm{G}^{\prime}}$ and $\omega_{\mathrm{G}^{\prime}}$. On the left side of the device we obtained a total of 1718 Raman spectra in two large areas $\approx 1 \mathrm{~mm}$ apart near the midline (areas 1 and 2 as seen in the inset of Fig. $4 \mathrm{~b})$. These data yield $\Gamma_{\mathrm{G}^{\prime}}=(34.7 \pm 4.6) \mathrm{cm}^{-1}$ and $\Gamma_{\mathrm{G}^{\prime}}=(37.3 \pm 5.2) \mathrm{cm}^{-1}$, with center positions $\omega_{\mathrm{G}^{\prime}}=(2735 \pm 4.3) \mathrm{cm}^{-1}$ and $\omega_{\mathrm{G}^{\prime}}=(2738 \pm 6.3) \mathrm{cm}^{-1}$, respectively for area 1 (purple) and area 2 (green). Additional Raman data were collected from eight widely distributed small areas (with less than 64 spectra per area) as shown in the inset of Fig. 4b (areas 3 to 10). While half of these areas (indicated in red) of sample B gave very homogeneous results, falling between the values for both $\omega_{\mathrm{G}^{\prime}}$ and $\Gamma_{\mathrm{G}^{\prime}}$ from the two large areas, the other half indicated in black show no significant overlap (see Supplement Table S1, S2), and gave mostly values of $\Gamma_{\mathrm{G}^{\prime}}$ greater than 40 $\mathrm{cm}^{-1}$. We have correlated this inhomogeneity observed in the Raman data from sample B with the positions of bilayer ribbons and buffer layer patches captured in confocal microscopy images in Fig. 3, and this will be explored in detail in a subsequent report. To summarize, in sample A we obtain narrower Raman G' linewidths with very little spread in the position, while in sample 
B we find similar results but with inhomogeneous regions indicated by the Raman data. Neither sample shows linewidths as broad as were seen for the EG sample made by our earlier synthesis method (Fig. S4f).

\section{RESULTS AND DISCUSSION}

Inhomogeneous transport characteristics may result from substrate topography, variation in EG layer number $[26,32,34,35]$, and/or the effect of strain [26-31,33,36]. Deconvolution of the effects of strain on transport in some EG samples can be problematic, as shown in our earlier work where EG was grown at a lower temperature of $1630{ }^{\circ} \mathrm{C}$ in Ar background without FTG on a substrate having a high miscut of $\approx 1.26^{\circ}$ relative to the $\mathrm{SiC}(0001)$ basal plane. Low-energy electron microscopy (LEEM) data confirmed monolayer graphene on step-bunched, parallel terraces of $0.5 \mu \mathrm{m}-2 \mu \mathrm{m}$ width, and Raman mapping provided correlated data covering the same region (see Supplement Fig. S4). The G' Raman band was fit to a Lorentzian function resulting in an average $G^{\prime}$ peak position $\omega_{G^{\prime}}=(2747.4 \pm 1.7) \mathrm{cm}^{-1}$ for the sampled region of diameter $44 \mu \mathrm{m}$. The small standard deviation indicated excellent homogeneity at the spatial resolution $(\sim 2 \mu \mathrm{m})$ under which the data was collected. However, the $\mathrm{G}^{\prime}$ linewidth of $\Gamma_{\mathrm{G}^{\prime}}=(63.8$ $\pm 2.6) \mathrm{cm}^{-1}$ was quite broad compared to that seen in graphene produced by exfoliation from graphite [32,36] and in our sample A and B. Magnetotransport measurements in small Hall bar devices made from this sample revealed low mobilities of $\mu<1000 \mathrm{~cm}^{2} / \mathrm{Vs}$ at $1.5 \mathrm{~K}$. While neither LEEM nor Raman captures the effects of terrace edges, the low mobility in this material may be linked to the broad, albeit uniform, G' linewidth. The large linewidth could result from inhomogeneous strain at sub-micron scale [36] that is averaged within the $1 \mu \mathrm{m}$ probe volume.

We correlate device transport in our FTG-grown EG with layer and strain homogeneity. Electrical measurements on samples A and B were made in a pumped liquid helium cryostat, and first will be correlated to our optical measurements, indicating the degree of inhomogeneous layer structure. Low-precision AC measurements were used to calculate the carrier density and mobility of the devices, and were repeated at various temperatures and current levels. We obtained precise QHE transport results for sample A with the original doping level obtained after removal of the $\mathrm{Pd} / \mathrm{Au}$ protective layer, at $n \approx 2.4 \times 10^{11} \mathrm{~cm}^{-2}\left(\mu \approx 4480 \mathrm{~cm}^{2} / \mathrm{Vs}\right)$. Fig. 5a shows magnetotransport characteristics for sample A at cryogenic temperatures. 
The hallmarks of the QHE in low-carrier-density EG are a broad plateau in the Hall resistance $R_{x y}$ with conventional value $\frac{h}{2 e^{2}}=12906.4035 \Omega$ (see Fig. 5a) and near-zero

231 longitudinal resistivity $\rho_{x x}$. The strength of this QHE plateau at high current and temperature is

232 enhanced at low perpendicular magnetic field strengths by the $\sqrt{ } B$ dependence of the Landau

233 level energies, and by field-dependent charge transfer from donors in the SiC substrate and

234 doping layer [8]. The presently used, commercial GaAs devices are rarely capable of sustaining 235 precise QHE measurements at currents above $0.2 \mathrm{~mA}$, and higher currents can exceed the range 236 of most state-of-the-art metrological instruments used to measure $R_{x y}$. For sample A, we used 237 two methods to obtain sensitive characterization of QHE device performance for higher current 238 levels, with the first based on the increase in longitudinal resistivity $\rho_{x x}$. Values of the 239 longitudinal resistivity $\rho_{x x}$ were measured at four temperatures between $1.6 \mathrm{~K}$ and $4.2 \mathrm{~K}$ over a 240 wide range of source-drain current (0.116 mA to $0.72 \mathrm{~mA})$, as shown in Fig. 5b. With an applied 241 field of $B=9 \mathrm{~T}$, zero dissipation is observed at $1.6 \mathrm{~K}$ for the full range of current up to $I_{x x} \approx$ $2420.72 \mathrm{~mA}$, and possibly at higher currents, but this could not be verified with the present 243 apparatus (Supplement Fig. S5a). These results exceed the highest critical currents reported to 244 date in graphene [9] (0.5 mA) or GaAs heterostructures [37] (0.6 mA). 

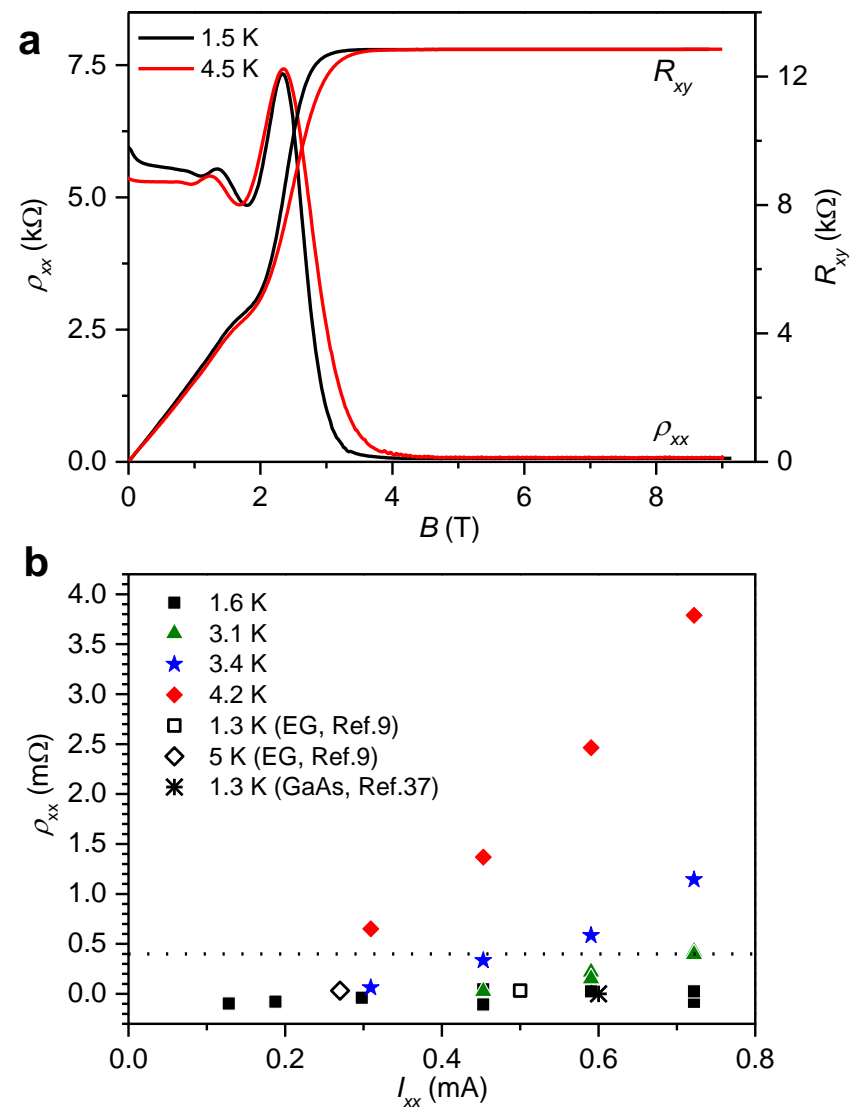

Figure 5. AC magnetotransport and DC precision measurements of $\rho_{x x}$ of sample A in a perpendicular magnetic field $B$. (a) Transport characteristics with $I_{x x}=1 \mu \mathrm{A}, n \approx 2.4 \times 10^{11} \mathrm{~cm}^{-2}$, and $\mu \approx 4480 \mathrm{~cm}^{2} / \mathrm{Vs}$ for sample A. (b) Precision measurements of $\rho_{x x}$ at $9 \mathrm{~T}$ as a function of source-drain DC current at $1.6 \mathrm{~K}$, 3.1 K, 3.4 K and 4.2 K. Dashed line at $\rho_{x x}=0.4 \mathrm{~m} \Omega$ indicates the degree of quantization sufficient to produce $R_{x y}$ values within five parts in $10^{9}$ of the ideal quantized value, as described in the text. Results of earlier high-current studies of the QHE in graphene devices and in GaAs are included for comparison.

Near the onset of thermally-activated dissipation [10,38], a linear relationship is generally observed between the deviation of the Hall resistance $\Delta R_{x y}$ from the quantized value $\left(\frac{h}{2 e^{2}}\right)$ and 254 the non-zero value of $\rho_{x x}$. A specialized two-terminal cryogenic current comparator (CCC) 255 bridge $^{39}$ was employed to measure values of $R_{x y}$ at $T=1.6 \mathrm{~K}$ and $T=4.2 \mathrm{~K}$ against a precision $256100 \mathrm{k} \Omega$ standard resistor at current levels of $0.3 \mathrm{~mA}, 0.45 \mathrm{~mA}, 0.6 \mathrm{~mA}$ and $0.72 \mathrm{~mA}$. While 257 GaAs-based QHE calibration of the $100 \mathrm{k} \Omega$ standard must be conducted at lower current levels and is thus less precise, the small differences measured for sample $\mathrm{A}$ at these two temperatures 
259

260

261

262

263

264

265

266

267

268

269

270

271

272

273

274

275

276

277

278

279

280

281

282

283

284

285

286

can be obtained with an uncertainty of better than $5 \times 10^{-9}$ in $R_{x y}$. Plotted against values of the longitudinal resistance $\rho_{x x}$ measured at $4.2 \mathrm{~K}$ for the same current levels, the deviation $\Delta R_{x y}=$ $R_{x y}(4.2 \mathrm{~K})-R_{x y}(1.6 \mathrm{~K})$ yields a slope $\Delta R_{x y} / \rho_{x x}(4.2 \mathrm{~K}) \approx 0.164 \pm 0.01$ (see Supplement Fig. S5b). Thus, the accuracy of the QHE is maintained at the level of $5 \times 10^{-9}$ in sample A up to $I_{x x} \approx$ $0.72 \mathrm{~mA}$ at $T=3.1 \mathrm{~K}$ and $9 \mathrm{~T}$, where $\rho_{x x} \leq 0.4 \mathrm{~m} \Omega$ is measured (as noted by the dashed line in Fig. 5b).

In our samples the adsorbed molecular doping layer acts as a gate, and it is possible to control the carrier concentration through this effect (see supplement, section 7). The concentration of adsorbed dopants was tuned after the initial fabrication of sample B, first by exposure to vapor from concentrated $\mathrm{HNO}_{3}$, followed by gentle heating of the sample in vacuum $[19,40]$. AC transport measurements were made on sample B at seven levels of carrier density, as shown in Fig. 6a and Fig. S7. Mobility in EG at low temperature is strongly dependent on carrier density, as demonstrated in sample B by the nearly inverse relationship between $\mu$ and $n$ for $\mathrm{n}$ type carrier concentrations below $3.0 \times 10^{11} \mathrm{~cm}^{-2}$. Mobility of $\mu \approx 3120 \mathrm{~cm}^{2} / \mathrm{Vs}$ is obtained in sample B at carrier density level of $n_{2} \approx 1.8 \times 10^{11} \mathrm{~cm}^{-2}$, compared to the mobility $\mu \approx 4480$ $\mathrm{cm}^{2} / \mathrm{Vs}$ at $n \approx 2.4 \times 10^{11} \mathrm{~cm}^{-2}$ for sample A.

Despite the presence of inhomogeneous strain and layer number in some areas of sample B, the measured mobility for $n_{0} \approx 0.9 \times 10^{10} \mathrm{~cm}^{-2}$ is $\mu \approx 18760 \mathrm{~cm}^{2} / \mathrm{Vs}$. This confirms that high mobility can be maintained in EG in the presence of localized defects [24] if the density of these defects is not too high, and indicates that a $10 \mathrm{~mm}^{2}$ area of sample B has very uniform carrier density at low temperature. For comparison, similar characteristics of $\mu$ and $n$ have been reported in gated, high-quality EG [33] devices of $<5 \mu \mathrm{m}$ width. For those, the mobility was somewhat higher at carrier density $n \approx 2 \times 10^{11} \mathrm{~cm}^{-2}$ and was seen to decrease for values of $n$ below $\approx 10^{11}$ $\mathrm{cm}^{-2}$, an effect that we did not observe in the FTG sample B for much lower carrier densities. Exfoliated graphene on h-BN [36] has exhibited mobilities up to four times what we measure at similar low carrier densities, and the authors did not report any decrease in the mobility at lower carrier density. More generally, the QHE in the millimeter-size EG samples described here far surpasses earlier results that we obtained using similar-scale CVD graphene [41]. 

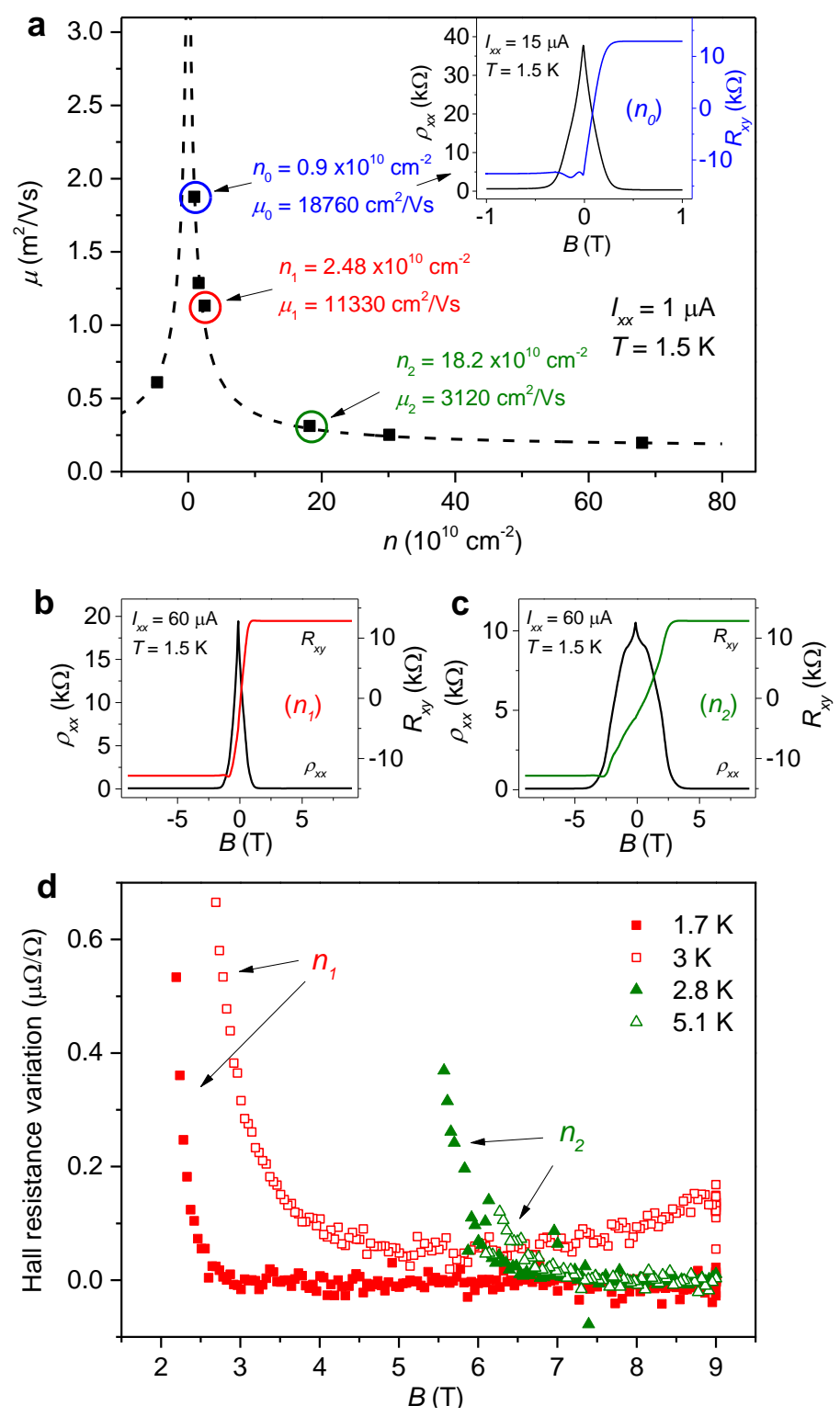

Figure 6. AC magnetotransport as a function of carrier density and DC precision QHE measurements of sample B. (a) Graph of mobility $\mu$ versus carrier density $n$ for sample B, calculated from the device conductivity $\sigma_{\mathrm{xx}}=e n \mu$ and the slope of the Hall resistance $R_{x y}(B)$. Starting at far left with low p-type doping $\left(4.7 \times 10^{10} \mathrm{~cm}^{-2}\right)$ changes in $n$ and $\mu$ were obtained by exposure to oxygen and heating the sample at increasing temperatures of $310^{\circ} \mathrm{C}-333{ }^{\circ} \mathrm{C}$ in vacuum. Fitting of the data to an inverse function $\mu=a+$ $b \times(n-c)^{-1}$ for $n>0$ results in the dotted curve, showing an approximate inverse relation, and this fit is mirrored about $n=0$ to show that the same relation may exist for $\mathrm{p}$-type carriers. Inset: transport characteristics for very low carrier density $\left(n_{0}\right)$ identified by the blue circle. (b) Sample B transport characteristics for low carrier density $\left(n_{1}\right)$. (c) Sample B transport characteristics for higher carrier density 
$\left(n_{2}\right)$. (d) Precise measurements of the deviation in the Hall resistance $R_{x y}(B)$ plotted for the two levels of carrier density $n_{1}$ and $n_{2}$. Data for $n_{1}$ was taken at $I_{\mathrm{xx}}=19.4 \mu \mathrm{A}$, while data for $n_{2}$ was taken at $I_{\mathrm{xx}}=116$ $\mu \mathrm{A}$. The dependence on $n$ and $T$ is described in the text.

Precise values of $R_{x y}$ in sample B were measured at doping levels indicated by $\left(n_{1}\right)$ and $\left(n_{2}\right)$ shown in Fig. 6a, with magnetotransport data given in Figs. 6(a-d). These measurements were made near the highest levels of current for which full quantization was maintained for each carrier density. At these current levels, $I_{\mathrm{xx}}\left(n_{1}\right) \approx 0.0194 \mathrm{~mA}$ and $I_{\mathrm{xx}}\left(n_{2}\right) \approx 0.116 \mathrm{~mA}$, conventional CCC measurements of $R_{x y}$ could be based on $100 \Omega$ standard resistors precisely calibrated against a NIST GaAs-based QHE standard. For the lower carrier density $n_{1}$ and $T \approx 1.7 \mathrm{~K}$, the value of $R_{x y}$ differed from $\frac{h}{2 e^{2}}$ by less than the measurement uncertainty of $\pm 5 \times 10^{-9}$ within the range $3 \mathrm{~T}<B<9 \mathrm{~T}$ (see Fig. 6d). For similar conditions but with $T=3.0 \mathrm{~K}$, the value measured for $R_{x y}$ differed from the ideal value by about five parts in $10^{8}$ near $B=6 \mathrm{~T}$, with increased variation from the ideal value at lower and higher field. When sample B was tuned to higher carrier density $\left(n_{2}\right)$, precise quantization was maintained over the range $7.5 \mathrm{~T}<B<9 \mathrm{~T}$ for higher measurement current of $I_{x x}=0.116 \mathrm{~mA}$ and $T=2.8 \mathrm{~K}$. For the same range of $B$ and $I_{x x}=$ $0.116 \mathrm{~mA}$, the value of $R_{x y}$ showed slight loss of quantization $\left(\Delta R_{x y} / R_{x y} \approx 1 \times 10^{-8}\right)$ for $T=5.1 \mathrm{~K}$ (Fig. 6d).

While our FTG graphene is produced on low-miscut $\mathrm{SiC}(0001)$ by annealing at $1900{ }^{\circ} \mathrm{C}$ in a near-equilibrium growth environment, improved QHE transport under relaxed conditions has also been reported in graphene which was grown on higher-miscut $\mathrm{SiC}(0001)$ by a hydrogensupported CVD process [9], where step bunching of SiC appears to be strongly suppressed. Stepbunching and bilayer regions are sources of scattering, and our results suggest that these regions may contribute to non-uniform strain in the EG layer. In semiconductor quantum Hall systems, the plateaus in $R_{x y}$ are centered at magnetic field values $B_{\mathrm{C}}=e n R_{H}$, where $n$ is the density of conducting electrons and $R_{H}$ is equal to $h / i e^{2}$ with $i$ taking on integer values, and the width of the plateau is related to the level of dissipation in the device. However, the $i=2$ plateau of epitaxial graphene can extend to very high values of $B$ because the charge transfer at the substrate/graphene interface is thought to be proportional to $B$ for some range of high magnetic field [8]. For carrier densities where the transition from the $i=6$ to $i=2$ plateau region is evident 
326 in EG magnetotransport, we can estimate the "half-width" of the $R_{x y}=\frac{h}{2 e^{2}}$ plateau by the

327 difference between the onset field $B_{0}$ and the field $B_{\mathrm{c}}$ when the extrapolated low field resistance

328 reaches $\frac{h}{2 e^{2}}, \Delta B=B_{c}-B_{0}$. The onset field $B_{0}$ is defined as the field where $R_{x y}\left(B_{0}\right)=0.99$

$329 R_{x y}\left(B_{\mathrm{c}}\right)$. The half-width $\Delta B$ is smaller in sample B than in sample A for similar levels of carrier

330 density $n \approx 2 \times 10^{11} \mathrm{~cm}^{-2}$ (see supplement Section 8), indicating that more dissipation is present

331 in sample B than in sample A for similar values of magnetic field and temperature, consistent

332 with the much lower critical current observed in sample B.

\section{CONCLUSION}

In conclusion, our observations for sample A clearly show that desirable magnetotransport properties are correlated with low and uniform strain in $\mathrm{EG}$ on $\mathrm{SiC}(0001)$ substrates. The uniform Raman G' band characteristics we observe compare favorably with those described in earlier reports [26-31] and provide support for the first example of EG with highly uniform strain at millimeter scale. The small G' FWHM and the narrow distribution of its position in sample A suggest reduced strain variation at the submicron scale [36]. In sample B, where bilayer inclusions are more common and strain is inhomogeneous in some areas, transport characteristics such as the lower critical current suggest greater dissipation, compared to sample A. However, our results indicate that the areas of homogeneous EG layer number and strain in sample B are sufficient for efficient millimeter-scale transport, as is evident from the QHE results over an extended range of magnetic field and by mobility exceeding $18700 \mathrm{~cm}^{2} /$ Vs for very low carrier density $n_{0} \approx 1 \times 10^{10} \mathrm{~cm}^{-2}$. While a better understanding of strain inhomogeneity in monolayer EG is still needed, our results indicate that uniform lattice strain and reduced topographic variation contribute to improved 2D quantized conductance at elevated current and temperature, and may provide direction for further advances in wafer-scale device fabrication.

\section{Methods}

Raman spectra were acquired under ambient conditions with a Renishaw InVia confocal Raman microscope equipped with $514.5 \mathrm{~nm}(2.41 \mathrm{eV})$ and $632.8 \mathrm{~nm}(1.96 \mathrm{eV})$ excitation lasers 
mapping measurements were performed using $514.5 \mathrm{~nm}$ excitation by raster scanning rectangular areas with a step size of $1 \mu \mathrm{m}$ and collecting the Raman $\mathrm{G}^{\prime}$ peak region with an exposure time of $10 \mathrm{~s}$ for each point. Raman maps were generated by fitting the spectra with a single Lorentzian peak and plotting the fitting parameters of FWHM and peak position at each pixel.

For initial transport characterization, four lock-in amplifiers monitored the longitudinal current $I_{x x}$ supplied at $13 \mathrm{~Hz}$ and three voltages developed in the device while we swept the perpendicular magnetic field strength $B$. We measured the Hall resistance $R_{x y}=V_{x y} / I_{x x}$ across the central pair of contacts (Fig S6). Longitudinal resistivity $\rho_{x x}$ was derived from the average resistance value measured across the other four symmetric contacts (4,6,10,12 in Fig S6), scaled by the ratio of width to length $(\alpha w / L=\alpha \times 5.6 \mathrm{~mm} / 1.8 \mathrm{~mm})$ separating these terminals, where $\alpha=$ 1.3 is a geometrical factor estimated from the DC transport simulation of a thin film conductor with the same semi-octagonal shape as our devices (Fig. S6). The high precision longitudinal resistivity was measured between the central pair of contacts $(5,11$ in Fig. S6) used to determine $R_{\mathrm{xy}}$ and one set of adjacent contacts, scaled by the ratio $\alpha w / L=\alpha \times 5.6 \mathrm{~mm} / 0.9 \mathrm{~mm}$, using a nanovolt meter (EM Electronics model N11) and recorded automatically using an Agilent 3458A DMM. Periodically reversed current was supplied by a battery-powered ramping voltage source $^{37}$. CCC measurements were made as described in Ref [39]. Estimation of carrier density and mobility were detailed in the supplement information, section 7 .

\section{Author Contributions}

Y.Y., G.C. and R.E.E. designed the experiments. Y.Y., G.C., C.C, C.-W.L., C.-I.L., P.M., I.G.C., R.M.F., G.R.J., R.M.F., and R.E.E. performed the experiments. Y.Y. and R.E.E. produced the samples and Y.Y fabricated the devices. Y.Y., G.C., A.R.H.W. and R.E.E. co-wrote the paper.

\section{Funding Sources}

The work of Y.Y. was supported by federal grant \#70NANB12H185.

\section{Notes}


379 Identification of commercial products or services used in this work does not imply endorsement

380 by the US government, nor does it imply that these products are the best available for the 381 applications described.

\section{ACKNOWLEDGMENT}

383 The work of C.C, C.-W.L. and C.-I.L at NIST was made possible by arrangement with Prof. C.384 T. Liang of National Taiwan University. We thank S. Lara-Avila of Chalmers University for 385 fabricating the transport device made of graphene grown at low temperature.

\section{REFERENCES}

[1] Geim, A. K. \& Novoselov, K. S. The rise of graphene. Nature Materials 6, 183-191 (2007)

388 [2] Novoselov, K. S., et al. A roadmap for graphene. Nature 490, 192-200 (2012).

389 [3] Forti, S. \& Starke, U. Epitaxial graphene on SiC: from carrier density engineering to quasi390 free standing graphene by atomic intercalation. J. Phys. D: Appl. Phys. 47, 094013 (2014).

391 [4] Varchon, F. et al. Electronic Structure of Epitaxial Graphene Layers on SiC: Effect of the 392 Substrate. Phys. Rev. Lett. 99, 126805 (2007).

393 [5] Fiori, G. et al. Electronics based on two-dimensional materials. Nature Nanotech. 9, 768-779 394 (2014).

395 [6] Grigorenko, A. N., Polini, M. \& Novoselov, K. S. Graphene plasmonics. Nature Photonics 6, 396 749-758 (2012).

397 [7] Bao, Q. and Loh, K.P., Graphene Photonics, Plasmonics, and Broadband Optoelectronic 398 Devices. ACS Nano 6, 3677-3694 (2012).

399 [8] Janssen, T. J. B. M. et al. Anomalously strong pinning of the filling factor $v=2$ in epitaxial 400 graphene. Phys. Rev. B 83, 233402 (2011). 
401 [9] Ribeiro-Palau, R., Lafont, R. F., Brun-Picard, J., et al. Quantum Hall resistance standard in 402 graphene devices under relaxed experimental conditions. Nature Nanotechnol. 10, 965 (2015).

403 [10] Jeckelmann, B \& Jeanneret, B. The quantum Hall effect as an electrical resistance standard. 404 Rep. Prog. Phys. 64 1603-1655 (2001).

405 [11] Virojanadara, C. et al. Homogeneous large-area graphene layer growth on 6H-SiC(0001). 406 Phys. Rev. B 78, 245403 (2008).

407 [12] de Heer, W.A. et al. Large area and structured epitaxial graphene produced by confinement 408 controlled sublimation of silicon carbide. Proc. Natl. Acad. Sci. USA., 108, 16900-16905 (2011).

409 [13] Tanaka, S., Morita, K. \& Hibino, H. Anisotropic layer-by-layer growth of graphene on 410 vicinal SiC(0001) surfaces. Phys. Rev. B 81, 041406 (2010).

411 [14] Norimatsu, W. \& Kusunoki, M. Formation process of graphene on SiC (0001). Physica E $412 \quad 42,691-694(2010)$.

413 [15] Nicotra, G. et al. Delaminated graphene at silicon carbide facets: atomic scale imaging and 414 spectroscopy. ACS Nano 7, 3045-3052 (2013).

415 [16] Goler, S. et al. Revealing the atomic structure of the buffer layer between $\mathrm{SiC}(0001)$ and 416 epitaxial graphene. Carbon 51, 249-254 (2013).

417 [17] Schardt J. et al. LEED structure determination of hexagonal $\alpha$-SiC surfaces. Surf. Sci. 337, $418 \quad 232-242(1995)$.

419 [18] Seyller, T. Passivation of hexagonal SiC surfaces by hydrogen termination, J. Phys.: 420 Condens. Matter 16, S1755-S1782 (2004).

421 [19] Yang, Y. et al. Low carrier density epitaxial graphene devices on SiC. Small 11, 90-95 422 (2015).

423 [20] Yager, T., et al. Express Optical Analysis of Epitaxial Graphene on SiC: Impact of 424 Morphology on Quantum Transport. Nano Lett. 13, 421 (2013). 
425 [21] Camara, N. et al. Anisotropic growth of long isolated graphene ribbons on the C face of 426 graphite-capped 6H-SiC. Phys. Rev. B 80, 125410 (2009).

427 [22] Real, M. A. et al. Graphene epitaxial growth on $\operatorname{SiC}(0001)$ for resistance standards. IEEE 428 Trans. Instrum. Meas. 62, 1454-1460 (2013).

429 [23] Yu, X. Z. et al. New synthesis method for the growth of epitaxial graphene. J. Electron 430 Spectros. Relat. Phenom. 184, 100-106 (2011).

431 [24] Dimitrakopoulos, C. et al. Effect of SiC wafer miscut angle on the morphology and Hall 432 mobility of epitaxially grown graphene. Appl. Phys. Lett. 98, 222105 (2011).

433 [25] Schumann, T. et al. Anisotropic quantum Hall effect in epitaxial graphene on stepped SiC 434 surfaces, Phys. Rev. B 85, 235402 (2012).

435 [26] Ni, Z. H. et al. Raman spectroscopy of epitaxial graphene on a SiC substrate. Phys. Rev. B 43677,115416 (2008).

437 [27] Röhrl, J. et al. Raman spectra of epitaxial graphene on SiC(0001). Appl. Phys. Lett. 92, $438201918(2008)$.

439 [28] Lee D. S. et al. Raman spectra of epitaxial graphene on SiC and of epitaxial graphene 440 transferred to $\mathrm{SiO}_{2}$. Nano Lett. 8, 4320-4325 (2008).

441 [29] Das, A., Pisana, S., Chakraborty, B. Monitoring dopants by Raman scattering in an 442 electrochemically top-gated graphene transistor. Nature Nanotechnol. 3, 210 (2008).

443 [30] Robinson, J. A. et al. Raman topography and strain uniformity of large-area epitaxial 444 graphene. Nano Lett. 9, 964-968 (2009).

445 [31] Robinson, J. A. et al. Correlating Raman spectral signatures with carrier mobility in 446 epitaxial graphene: a guide to achieving high mobility on the wafer scale. Nano Lett. 9, 2873$4472876(2009)$. 
448 [32] Neumann, C. et al. Raman spectroscopy as probe of nanometre-scale strain variations in 449 graphene. Nature Comm. 6, 8429 (2015).

450 [33] Farmer, D. B. et al. Charge trapping and scattering in epitaxial graphene. Phys. Rev. B 84, $451205417(2011)$.

452 [34] Yakes, M. K. et al. Conductance Anisotropy in Epitaxial Graphene Sheets Generated by 453 Substrate Interactions. Nano Lett. 10, 1559-1562 (2010).

454 [35] Ji, S.-H. et al. Atomic-scale transport in epitaxial graphene. Nature Mat. 11, 114-119 455 (2012).

456 [36] Couto, Nuno J. G., et al. Random strain fluctuations as dominant disorder source for high457 quality on-substrate graphene devices. Phys. Rev. X 4, 041019 (2014).

458 [37] Jeckelmann, B., Jeanneret, B., and Inglis, D. High-precision measurements of the quantized 459 Hall resistance: experimental conditions for universality. Phys. Rev. B 55, 13124 (1997).

460 [38] F. Schopfer and W. Poirier. Quantum resistance standard accuracy close to the zero461 dissipation state. J. Appl. Phys. 114, 064508 (2013).

462 [39] Hernandez-Marquez, F. L. et al. Precision high-value resistance scaling with a two-terminal 463 cryogenic current comparator. Rev. Sci. Instrum. 85, 044701 (2014).

464 [40] Chieh-I Liu, et al. Charge trapping in monolayer and multilayer epitaxial graphene, $J$. $465 \quad$ Nanomaterials 2016, Article ID 7372812 (2016).

466 [41] Shen, T.et al., Quantum Hall effect on centimeter scale chemical vapor deposited graphene 467 films. Appl. Phys. Lett. 99, 232110 (2011). 